\title{
Patterns of Cerebral Glucose Metabolism Detected with Positron Emission Tomography Differ in Multiple System Atrophy and Olivopontocerebellar Atrophy
}

\author{
Sid Gilman, MD, ${ }^{*}$ Robert A. Koeppe, PhD, $\nmid$ Larry Junck, MD, ${ }^{*}$ Karen J. Kluin, MS,$* \ddagger$ \\ Mary Lohman, BA,* and Roy T. St Laurent, PhD $\$$
}

\begin{abstract}
We used positron emission tomography with $\left[{ }^{18} \mathrm{~F}\right]$ fluorodeoxyglucose to study local cerebral metabolic rates for glucose (ICMRgIc) in patients with multiple system atrophy (MSA), sporadic olivopontocerebellar atrophy (sOPCA), and dominantly inherited olivopontocerebellar atrophy (DOPCA) in comparison with normal control subjects. In MSA, absolute ICMRglc was significantly decreased in the brainstem, cerebellum, putamen, thalamus, and cerebral cortex. In SOPCA, absolute ICMRglc was significantly decreased in the brainstem, cerebellum, putamen, thalamus, and cerebral cortex. In dOPCA, absolute ICMRglc was significantly decreased in the brainstem and cerebellum but not in the other structures. Examination of ICMRglc normalized to the cerebral cortex in comparison with normal controls revealed in MSA significant decreases in the brainstem, cerebellum, and putamen but, in both SOPCA and dOPCA, significant decreases only in the brainstem and cerebellum. The findings indicate that these three disorders all show a marked decrease of ICMRglc in the brainstem and cerebellum but differ in the degree of hypometabolism in forebrain and cerebral cortical structures. The results are consistent with the possibility that, in many cases, sOPCA will evolve into MSA. Moreover, positron emission tomography may provide helpful diagnostic information in these neurodegenerative diseases.
\end{abstract}

Gilman S, Koeppe RA, Junck L, Kluin KJ, Lohman M, St Laurent RT. Parterns of cerebral glucose metabolism detected with positron emission tomography differ in multiple system atrophy and olivopontocerebellar atrophy. Ann Neurol 1994;36:166-175

Multiple system atrophy (MSA) is an adult-onset sporadic neurodegenerative disease presenting clinically with symptoms and signs of extrapyramidal, cerebellar, and autonomic dysfunction in various combinations [1-5]. The extrapyramidal symptoms are poorly responsive or unresponsive to levodopa therapy $[6,7]$. The neuropathological features of MSA consist of degenerative changes, including neuronal loss and gliosis, in the basal ganglia (putamen and globus pallidus), brainstem and cerebellum (substantia nigra, locus ceruleus, dorsal vagal nuclei, vestibular nuclei, inferior olives, pontine nuclei, and cerebellar Purkinje cells), and spinal cord (pyramidal tracts, intermediolateral columns, and Onuf's nuclei) $[2,4,8]$. Other central nervous system structures that can be affected include the optic nerves, cerebral cortex, caudate nuclei, subthalamic nuclei, red nuclei, dentate nuclei, anterior horn cells, and sympathetic ganglia [2, 4].
Several neurological diseases are considered to be subgroups of MSA, including striatonigral degeneration (SND) [9-12], Shy-Drager syndrome (SDS) [1, 3, 13-15], and olivopontocerebellar atrophy (OPCA) with autonomic insufficiency or extrapyramidal disorders $[2,16-23]$. When detected without autonomic insufficiency or cerebellar disorder, SND is considered to be "possible MSA," whereas SDS and OPCA with autonomic insufficiency or extrapyramidal disorders are considered to be "probable MSA" [4]. In SND the neuropathological changes affect chiefly the basal ganglia and substantia nigra, in SDS the brainstem and spinal cord, and in OPCA the inferior olives, pons, and cerebellum.

There is substantial overlap in symptoms, signs, and neuropathological changes in SND, SDS, and OPCA and thus strong justification for considering them all potentially as forms of MSA [4]. Some patients with
From the Departments of *Neurology, Internal Medicine, and $\ddagger$ Physical Medicine and Rehabilitation, University of Michigan Medical Center, and \$Department of Biostatistics, University of Michigan School of Public Health, Ann Arbor, MI.
Received Apr 19, 1993, and in revised form Dec 27. Accepted for publication Jan 6, 1994.

Address correspondence to Dr Gilman, Department of Neurology, University of Michigan Medical Center, 1500 E. Medical Center Dr, Ann Arbor, MI 48109-0316. 
MSA initially develop extrapyramidal symptoms and later experience autonomic or cerebellar symptoms or both [4]. Others first manifest cerebellar symptoms and later develop autonomic or extrapyramidal symptoms. Although signs of cerebellar dysfunction may not be evident in patients who develop SND or SDS, neuropathological studies demonstrate degenerative changes in the cerebellum and brainstem in most cases [8]. The loss of cerebellar and brainstem neurons is greatest in patients with MSA presenting initially with cerebellar symptoms, less in those with SDS, and least in those with SND [8]. In some patients, especially those with hereditary disease, OPCA occurs without involving the extrapyramidal or autonomic systems. In others, mostly in sporadic cases, OPCA can progress to MSA. Currently no method is available to determine whether sporadic OPCA will progress to include other manifestations of MSA.

Previously, using $\left[{ }^{18} \mathrm{~F}\right]$ fluorodeoxyglucose (FDG) and positron emission tomography (PET), we demonstrated hypometabolism in the cerebellum and brainstem of patients with hereditary and sporadic OPCA [24]. The degree of hypometabolism was directly related to the severity of the ataxia and to the degree of atrophy of these structures in anatomical imaging studies [24-26]. The present study was undertaken to examine local cerebral metabolic rates for glucose (ICMRglc) in the central nervous system of patients with MSA in comparison with patients with OPCA who presented with no clinical evidence of involvement of autonomic or extrapyramidal systems, and with normal control subjects similarly distributed by age and sex. We separated the OPCA parients into two groups, those with sporadic disease (sOPCA) and those with disease inherited in an autosomal dominant pattern (dOPCA). This separation was made because the dOPCA group should constitute a group of pure OPCA patients, that is, patients without involvement of multiple systems, since people with dOPCA generally do not exhibit extrapyramidal or autonomic symptoms. The sOPCA group may include patients with clinical symptoms only of OPCA but with pathological involvement in multiple systems and with a metabolic pattern similar to that seen in MSA, but the dOPCA group should be free of such patients.

The hypothesis underlying this project was that the metabolic profile should be different in the three groups (MSA, sOPCA, and dOPCA). Based upon the sites of degeneration in neuropathological studies, we anticipated finding hypometabolism in the basal ganglia, cerebellum, and brainstem in MSA, but only in the cerebellum and brainstem in dOPCA. Many patients with sOPCA progress to MSA, and thus we anticipated finding some degree of hypometabolism in the basal ganglia, reflecting subclinical disease, in addition to involvement of the cerebellum. A preliminary communication concerning this study has been published [27].

\section{Materials and Methods \\ Patient Groups and Normal Subjects}

The studies were approved by the Institutional Review Board, and informed consent was obtained from each patient and normal control subject. The patients were segregated into three groups based upon the history and physical findings, i.e., multiple system atrophy (MSA), sporadic olivopontocerebellar atrophy (sOPCA), and dominant olivopontocerebellar atrophy (dOPCA) (Table 1). We studied 126 subjects ( 51 normal controls and 75 patients), age $51 \pm 16$ years (mean $\pm \mathrm{SD}$ ), including 59 men (19 normal controls and 40 patients), age $52 \pm 15$ years, and 67 women (32 normal controls and 35 patients), age $50 \pm 16$ years. Significant differences in age were found among the four diagnosis groups $(p<0.01)$ with a one-way analysis of variance (ANOVA). Three patients, two with SOPCA and one with dOPCA, were included in our previous studies [24-26], but all of these patients were rescanned for the present study. One of the patients with SOPCA in the previous communications developed extrapyramidal and autonomic symptoms and signs subsequent to the initial study and is now in the group of MSA patients.

In analyzing the duration of symptoms, we found that skewness in the distribution precluded reporting means and standard deviations. Accordingly, we report the range and median duration in each group. For MSA the range of duration was from 2 to 27 years with a median of 5 years. For sOPCA, the range was from 2 to 24 years with a median of 4 years, and for dOPCA the range was from 2 to 50 years

Table 1. Numbers, Ages (Mean \pm SD), and Sex Distributions of the Subjects Studied

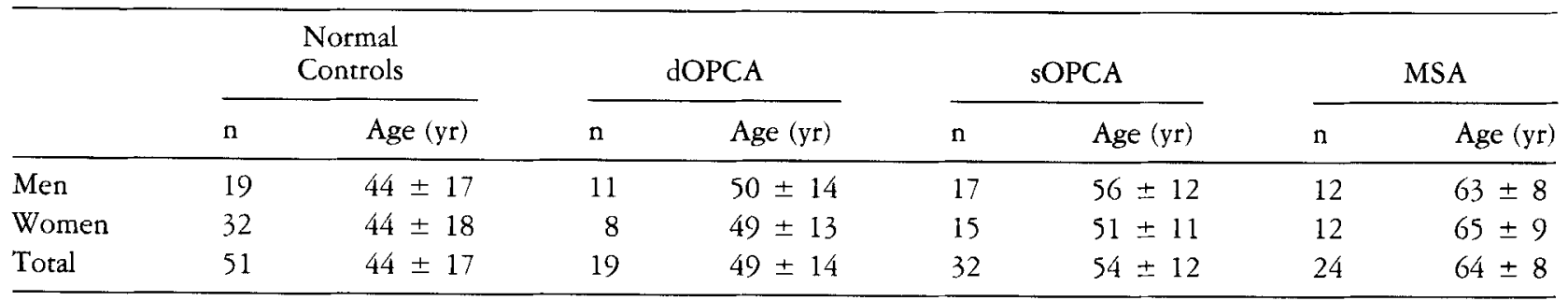

$\mathrm{dOPCA}=$ dominantly inherited olivopontocerebellar atrophy; sOPCA = sporadic olivopontocerebellar atrophy; MSA $=$ multiple system atrophy. 


\begin{tabular}{|c|c|c|c|c|c|c|c|c|c|c|c|c|}
\hline & \multicolumn{12}{|c|}{ Patient No. } \\
\hline & 1 & 2 & 3 & 4 & 5 & 6 & 7 & 8 & 9 & 10 & 11 & 12 \\
\hline Sex & $\mathrm{F}$ & $\mathrm{M}$ & $\mathbf{F}$ & $\mathrm{F}$ & $M$ & $\mathrm{~F}$ & $\mathrm{~F}$ & $\mathrm{~F}$ & $\mathbf{M}$ & $\mathrm{F}$ & M & M \\
\hline Age (yr) & 59 & 61 & 65 & 82 & 68 & 67 & 58 & 65 & 49 & 76 & 58 & 62 \\
\hline $\begin{array}{l}\text { Duration of } \\
\text { symptoms } \\
\text { (yr) }\end{array}$ & 4 & 7 & 4 & 3 & 11 & 9 & 2 & 6 & 2 & 5 & 4 & 4 \\
\hline Akinesia & ++ & ++ & +++ & + & ++ & - & + & +++ & ++ & +++ & ++ & ++ \\
\hline Rigidity & ++ & +++ & $+t+$ & ++ & ++ & - & ++ & +++ & $+t$ & +++ & ++ & $+t$ \\
\hline $\begin{array}{l}\text { Tremor (extra- } \\
\text { pyramidal) }\end{array}$ & - & - & - & - & - & - & - & + & + & + & - & - \\
\hline $\begin{array}{l}\text { Hypokineric } \\
\text { speech }\end{array}$ & ++ & $+t$ & + & - & ++ & - & + & ++ & $+t$ & ++ & + & $+t$ \\
\hline $\begin{array}{c}\text { Response to } \\
\text { levodopa }\end{array}$ & $\mathrm{NT}$ & - & - & $\mathrm{NT}^{2} \mathrm{~T}$ & - & NT & NT & - & ++ & - & - & - \\
\hline $\begin{array}{c}\text { Ocular dys- } \\
\text { metria }\end{array}$ & + & + & + & + & ++ & + & + & - & - & ++ & ++ & + \\
\hline Gait ataxia & + & + & + & $+t$ & +++ & +++ & ++ & - & - & +++ & +++ & +++ \\
\hline Limb ataxia & + & - & + & - & ++ & ++ & + & - & - & +++ & $+t$ & + \\
\hline Ataxic speech & + & + & + & ++ & ++ & ++ & + & + & + & + & $+t+$ & ++ \\
\hline Spastic speech & - & + & + & + & + & + & + & ++ & + & + & + & + \\
\hline $\begin{array}{l}\text { Extensor plan- } \\
\text { tar signs }\end{array}$ & - & - & - & - & ++ & ++ & ++ & ++ & - & ++ & ++ & - \\
\hline $\begin{array}{l}\text { Postural hypo- } \\
\text { tension }\end{array}$ & ++ & ++ & $+t$ & - & +++ & +++ & ++ & ++ & ++ & - & - & - \\
\hline $\begin{array}{l}\text { Urinary incon- } \\
\text { tinence }\end{array}$ & ++ & +++ & - & - & +++ & $+t+$ & ++ & ++ & + & + & + & - \\
\hline $\begin{array}{l}\text { MRI } \\
\text { Cerebral at- } \\
\text { rophy }\end{array}$ & + & + & + & - & ++ & + & - & - & - & + & + & + \\
\hline $\begin{array}{c}\text { Cerebellar } \\
\text { atrophy }\end{array}$ & ++ & - & ++ & - & $+t$ & ++ & ++ & - & - & ++ & + & ++ \\
\hline
\end{tabular}

$(-)=$ absent; $(+)=$ mild; $(++)=$ moderate; $(+++)=$ marked; NT $=$ not tested; MRI = magnetic resonance imaging.

with a median of 10 years. Studies were performed on normal control subjects who had no history of neurological disorder and had no abnormalities on general physical and neurological examinations (see Table 1). At the time of the PET study, none of the patients or normal control subjects were taking medications that are known to influence cerebral metabolism. Four of the 24 patients with MSA were taking medications for parkinsonism (carbidopa/levodopa: 25:100 four times daily, 25:100 three times daily, 10:100 four times daily along with selegeline $5 \mathrm{mg}$ twice daily, and 10:100 three times daily).

We studied 23 patients with probable MSA and 1 patient (patient 16) with possible MSA [4]. The diagnosis of probable MSA was based upon demonstration of clinical signs of at least two of the following: (1) extrapyramidal disorder, (2) autonomic failure, and (3) cerebellar dysfunction. For the demonstration of extrapyramidal disorder, we required at least two of the following signs: akinesia, rigidity, tremor, and hypokinetic speech. In most cases we also assessed the degree of responsiveness to levodopa with clinical neurological evaluations before and after treatment with carbidopal levodopa at a dose of $25: 250$, gradually increased from one to four tablets daily. Patients were judged to be unresponsive or mildly, moderately, or markedly responsive. Seventeen patients were tested; 14 were unresponsive, 1 was mildly responsive, and 2 were moderately responsive (Table 2 ). The remaining 7 patients could not be tested.
The demonstration of changes in the putamen in magnetic resonance imaging (MRI) scans [28, 29] provided helpful information for the diagnosis of MSA but was not required since these changes are not specific to MSA [30]. For the demonstration of cerebellar dysfunction, we required at least two signs, including limb and/or gait ataxia along with ocular dysmetria or ataxic speech. For the demonstration of autonomic failure, we required postural hypotension or urinary incontinence without obstruction. The criteria for postural hypotension were similar to those of McLeod and Tuck [31], including an orthostatic drop of $30 \mathrm{~mm}$ or more in systolic blood pressure and $20 \mathrm{~mm}$ or more in diastolic blood pressure with an increase in heart rate of no more than 10 beats per minute. Blood pressure and pulse were measured supine and again 2 minutes after the patient had assumed the standing position. In patients who could not tolerate standing for 2 minutes, these changes were accepted after the patient stood as long as tolerated. Blood pressure measurements were made when the patients had been withdrawn from treatment with any medication that might induce postural hypotension. Most of the patients with postural hypotension had an associated history of sexual impotence (in the males), anhydrosis, or incontinence without outflow obstruction. Some of the patients without postural hypotension had incontinence without outflow obstruction, and all of these patients who were males had sexual impotence. In Table 2, the degree of postural hypotension and urinary incontinence refers to 


\begin{tabular}{|c|c|c|c|c|c|c|c|c|c|c|c|}
\hline \multicolumn{12}{|c|}{ Patient No. } \\
\hline 13 & 14 & 15 & 16 & 17 & 18 & 19 & 20 & 21 & 22 & 23 & 24 \\
\hline $\mathbf{F}$ & $\mathbf{M}$ & $\mathbf{F}$ & $\mathbf{M}$ & $\mathrm{F}$ & $\mathrm{M}$ & $\mathrm{F}$ & M & $\mathrm{F}$ & $\mathrm{M}$ & M & M \\
\hline 70 & 73 & 51 & 72 & 65 & 50 & 67 & 64 & 55 & 71 & 61 & 61 \\
\hline 5 & 4 & 2 & 10 & 4 & 20 & 3 & 16 & 3 & 5 & 2.5 & 7 \\
\hline$++t$ & ++ & $+t+$ & + & +++ & ++ & +++ & ++ & + & + & - & + \\
\hline$+t+$ & + & ++ & + & +++ & - & +++ & ++ & + & ++ & + & +++ \\
\hline+ & + & - & + & - & ++ & - & + & + & - & - & + \\
\hline+++ & + & ++ & ++ & +++ & - & + & ++ & - & + & + & ++ \\
\hline- & - & - & - & + & $\mathrm{NT}$ & - & ++ & NT & - & NT & - \\
\hline+ & - & + & + & + & + & + & + & + & - & + & + \\
\hline- & - & ++ & - & $++t$ & + & - & - & ++ & + & ++ & $+t+$ \\
\hline- & - & +++ & - & + & ++ & - & - & +++ & + & +++ & ++ \\
\hline+ & - & + & - & + & +++ & + & - & +++ & ++ & ++ & $+t+$ \\
\hline++ & - & + & + & ++ & - & ++ & + & + & + & + & ++ \\
\hline- & - & - & - & + & - & - & - & + & - & - & + \\
\hline- & ++ & +++ & - & $++t$ & ++ & +++ & ++ & +++ & + & - & + \\
\hline+ & - & $+t+$ & - & +++ & $+t+$ & - & + & $+t+$ & ++ & - & + \\
\hline++ & - & - & - & - & - & - & - & - & - & - & - \\
\hline++ & - & +++ & - & ++ & + & - & - & ++ & ++ & +++ & $+t+$ \\
\hline
\end{tabular}

the severity of the symptoms from the patients' perspective. Utilizing the criteria defined above, 13 of the patients had extrapyramidal, cerebellar, and autonomic dysfunction, 6 had extrapyramidal and autonomic, 3 had extrapyramidal and cerebellar, 1 had cerebellar and autonomic, and 1 had extrapyramidal alone ("possible MSA") (see Table 2).

The diagnosis of OPCA was based upon a history of progressive deterioration in cerebellar function manifested by ataxia of gait and speech, usually accompanied by ataxia of ocular and limb movements, in the absence of a disorder of sensory function sufficient to cause ataxia, medications (e.g., phenytoin), toxins (e.g., alcohol), evidence of a neoplasm in the cerebellum or elsewhere, or evidence of multiple sclerosis or other diseases that can cause progressive cerebellar ataxia. The diagnosis was assisted by the demonstration of cerebellar and brainstem atrophy in computed tomographic (CT) or MRI scans, but this was not required since OPCA can occur in the absence of atrophy in anatomic imaging studies [24].

Each patient was evaluated with a complete history, physical examination, neurological examination, laboratory tests to exclude other diseases, and MRI imaging to exclude demyelinative disease and other structural abnormalities. Speech was evaluated as described previously $[26,32\}$. Laboratory tests included a complete blood count, serum profiles of hepatic and renal function, brainstem auditory, visual, and somatosensory evoked potentials, serum levels of vitamins $E$ and $B_{12}$ and folic acid, and studies of thyroid function. In patients with symptoms for less than 3 years, a search was made for an occult malignancy, including breast and pelvic examinations in women, prostate examination in men, acid phosphatase and prostate specific antigen levels, stool guaiac tests for occult blood and chest $\mathrm{x}$-rays. In addition, anti-Purkinje cell antibodies were sought in blood samples for patients with ataxia of less than 3 years' duration. All patients were evaiuated with MRI to determine the extent of volume loss of the structures under study. The diagnosis of sOPCA was made if there was no family history of a similar illness after a detailed history had been taken with construction of a family tree. The diagnosis of dOPCA was based upon a clear family history of a similar disorder in a parent. Patients with a family history suggesting autosomal recessive inheritance were excluded from this study.

The neurological examinations were conducted by a neurologist (S.G.) blinded to the PET data. Speech was evaluated by a speech-language pathologist (K.J.K.) also blinded to the results of the PET studies. The severity of the clinical neurological features in the patients with MSA was graded on the scales shown in Table 2.

\section{Positron Emission Tomography Studies}

These studies were performed with the normal control subjects and the patients lying supine and awake in a quiet room with eyes open from 5 minutes before injection until completion of the scan. To ensure that similar levels and angles of scanning were obtained, all subjects were aligned parallel to 
the canthomeatal (CM) line with laser beams. Scans were performed 30 to 90 minutes after intravenous injection of $10 \mathrm{mCi}$ of FDG, which was synthesized by an adaptation of the method of Hamacher and colleagues [33].

PET images were acquired with a Siemens/CTI 931/08-12 scanner, which has an intrinsic in-plane resolution of $5.5-\mathrm{mm}$ full-width-at-half-maximum (FWHM) and a $z$-axis resolution of 7.0-mm FWHM. Fifteen planes with 6.75 -mm center-tocenter separation were imaged simultaneously. Two sets of interleaved planes were acquired, yielding 30 planes, each separated by $3.375 \mathrm{~mm}$. Attenuation correction was calculated by fitting ellipses to the scalp contour.

Blood samples were collected from the radial artery for estimation of the arterial radioactivity input to brain. Local cerebral metabolic rates for glucose (ICMRglc) were calculated with a three-compartment model and a single scan approximation [34]. Volumes-of-interest (VOIs) were studied in the brainstem, cerebellar vermis, cerebellar hemispheres, thalamus, basal ganglia (caudate nucleus and putamen), and cerebral cortex. Levels comprising each VOI were contiguous. PET images were viewed in the transverse and sagittal planes. Data were collected from transverse sections with a 2.6- $\mathrm{cm}^{2}$ polygon over each cerebellar hemisphere from 4 of the 30 levels, a $1.3-\mathrm{cm}^{2}$ parallelogram over each thalamus from 2 of the 30 levels, a $0.9-\mathrm{cm}^{2}$ square over each caudate nucleus from 2 of the 30 levels, and a $1.9-\mathrm{cm}^{2}$ parallelogram over each putamen from 2 of the 30 levels. Data were collected in the midsagittal plane from the pons with a $1.3 \times$ $1.5 \times 1.1-\mathrm{cm}$ right parallelepiped and from the superior and inferior parts of the cerebellar vermis with a $1.3 \times 1.9 \times$ $1.1-\mathrm{cm}$ right parallelepiped. Each VOI was automatically centered over a local peak in ICMRglc. VOIs from the cerebellar vermis were posterior to the fourth ventricle. The brainstem VOI chiefly reflects the pons, but the mesencephalon could be partially represented.

Data from the cerebral cortex were obtained in transverse images by measuring ICMRglc in the cortical ribbon from 8 or 10 consecutive planes of 30 , beginning with the plane containing the most superior portion of the cingulate gyrus and continuing inferiorly to the plane containing the lowest portion of the thalamus. This was accomplished with an algorithm that detects the outer edge of the cortical rim from an image that has been passed through a contrast-enhancing filter. The algorithm then identifies on the original image a cortical band that extends inward from this edge until either the metabolic rate drops below the value on the outer edge of the rim or the band reaches a width of $15 \mathrm{~mm}$. An areaweighted mean metabolic rate was computed for the mean cortical value. Normalized values were obtained by dividing individual VOI values by the mean value from the cerebral cortex over all slices. Data were normalized to determine whether changes in absolute values reflect the effects of the diseases upon specific structures or upon the brain as a whole. The cerebral cortex was selected for normalizing the data because it is a large structure not known previously to be involved in these disorders.

\section{Data Analysis}

A data analyst (M.L.) analyzed VOIs in the PET studies and these data were shared among the investigators only after completion of measurements from all studies. Absolute and normalized regional glucose cerebral metabolic rates were analyzed using multivariate repeated measures analysis of covariance and univariate analysis of covariance (ANCOVA). Absolute metabolic rates were analyzed from the following seven brain regions: brainstem, cerebellar vermis, cerebellar hemispheres, caudate nuclei, putamen, thalamus, and cerebral cortex. Normalized metabolic rates were analyzed for the first six of these regions.

In the multivariate analysis, the dependent variable was regional brain metabolic activity (either absolute or normalized). Measurements on seven (for absolute data) or six (for normalized data) brain regions constituted the repeated measurements on each patient. The primary independent variable in the analysis was the categorical variable diagnosis group (MSA, sOPCA, dOPCA, or normal control). In addition, the factor sex and the continuous covariate age were included in the model. All possible interactions between diagnosis group, sex, and age were considered. Age was standardized by subtracting the mean age across all subjects.

The data were analyzed in several stages. In the first stage, not presented here, plots of the data were used to assess gross trends and relationships between variables. Second, a repeated measures ANOVA model was fitted to the data incorporating region as the repeated (within-subject) measures variable, diagnosis group, sex, and age, and all of the interactions among these variables to determine whether differences existed between brain regions. Dependent upon the outcome of this stage, a univariate ANOVA or ANCOVA model was developed to examine each region separately. The factors incorporated into the univariate ANOVA model were those identified in the second stage as having an overall effect on glucose metabolic rate across regions or having a differential effect on glucose metabolic rates between regions. In the last stage, comparisons of individual diagnosis group means were made, where appropriate.

For all pairwise differences, a Tukey-Kramer multiple comparison adjustment [35] was used. Hence, the reported $p$ values incorporate this adjustment. When the final model included age, diagnosis group means were appropriately adjusted for age. Data analysis was performed with the SAS statistical package (SAS Institute Inc., Cary, NC).

\section{Results}

\section{Analysis of Absolute Levels of ICMRglc}

Repeated measures ANCOVA revealed highly significant differences between brain regions $(p<$ 0.0001 ) and strong evidence for differences in the effects of diagnosis group between regions $(p<0.0001)$. Evidence was found also for differences in region by age $(p<0.005)$. No evidence was adduced for differences in region by sex, nor for differences between regions in interaction effects between age, sex, or diagnosis group. Examination of the overall effects of diagnosis, age, and sex (looking across all regions simultaneously) indicated large differences between diagnosis groups $(p<0.0001)$ but no other significant effects.

The significant effects found between regions, and differences in the effects of diagnosis groups, indicate 
that differences in metabolic rates between diagnosis groups must be examined for each region individually. The evidence for a difference in age effect by region requires that this variable be incorporated in the univariate analysis as a covariate.

Separate univariate ANCOVA analyses for each region (including the factors diagnosis and age) revealed that age effects were significant in the caudate nucleus $(p=0.0003)$, putamen $(p=0.021)$, and cerebral cortex $(p=0.0008)$. In the thalamus there was some indication of a trend with age $(p=0.06)$. No evidence was found in any of the remaining regions for an age effect ( $p>0.10$ in each). To provide a common basis for comparison, however, subsequent analysis of absolute metabolic rates by diagnosis group incorporated an adjustment for age.

Very strong evidence was found for overall differences in diagnosis groups in the brainstem, cerebellar vermis, cerebellar hemispheres, and putamen $(p<$ 0.0001 in each). Strong evidence for overall differences in diagnosis groups was also found in the thalamus $(p=0.0016)$ and cerebral cortex $(p=0.004)$. Evidence for a difference in caudate nucleus was also found $(p=0.041)$. To investigate these differences further, pairwise comparisons among mean ICMRglc values were examined.

After controlling for multiple comparisons, we found that in MSA, absolute ICMRglc was markedly and significantly decreased relative to normals in the brainstem, cerebellar vermis, cerebellar hemispheres, and putamen $(p<0.0001$ in each) and also in the thalamus and cerebral cortex ( $p<0.05$ in each) (Table 3 , Fig). In sOPCA, ICMRglc was decreased in the brainstem, cerebellar vermis and cerebellar hemispheres $(p<0.0001$ in each), and putamen $(p<0.01)$, but somewhat less in the cerebral cortex and thalamus $(p<0.05)$. In dOPCA, lCMRglc was greatly decreased in the brainstem, cerebellar vermis, and cerebellar hemispheres $(p<0.0001$ in each), but no other regions showed significant decreases. Statistically significant differences between MSA and DOPCA were found in the putamen $(p=0.02)$ only.

\section{Analysis of ICMRglc Normalized \\ to the Cerebral Cortex}

Repeated measures ANCOVA revealed highly significant differences between brain regions $(p<$ $0.0001)$ and strong evidence for differences in the effects of diagnosis group between regions $(p<0.0001)$. Evidence was found also for differences in region by age $(p<0.02)$. No evidence was adduced for differences in the effects of region by sex, nor for differences between regions in interaction effects between age, sex, or diagnosis group. Examination of the overall effects of diagnosis, age, and sex (looking across all regions simultaneously) indicated strong differences be-
Table 3. Absolute Local Cerebral Metabolic Rates for Glucose

\begin{tabular}{lllll} 
& $\begin{array}{l}\text { Normal } \\
\text { Controls } \\
(\mathrm{n}=51)\end{array}$ & $\begin{array}{l}\text { dOPCA } \\
(\mathrm{n}=19)\end{array}$ & $\begin{array}{l}\text { sOPCA } \\
(\mathrm{n}=32)\end{array}$ & $\begin{array}{l}\text { MSA } \\
(\mathrm{n}=24)\end{array}$ \\
\hline $\begin{array}{l}\text { Brainstem } \\
\begin{array}{l}\text { Cerebellar } \\
\quad \text { vermis }\end{array}\end{array}$ & 5.98 & $4.74^{\mathrm{a}}$ & $4.50^{\mathrm{a}}$ & $4.08^{\mathrm{a}}$ \\
$\begin{array}{l}\text { Cerebellar } \\
\quad \text { hemispheres }\end{array}$ & 6.71 & $5.17^{\mathrm{a}}$ & $4.42^{\mathrm{a}}$ & $4.37^{\mathrm{a}}$ \\
$\begin{array}{l}\text { Thalamus } \\
\text { Caudate nuclei }\end{array}$ & 8.67 & $4.76^{\mathrm{a}}$ & $4.24^{\mathrm{a}}$ \\
$\begin{array}{l}\text { Putamen } \\
\text { Cerebral cortex }\end{array}$ & 6.88 & $8.27^{\mathrm{ns}}$ & $7.81^{\mathrm{d}}$ & $7.39^{\mathrm{c}}$ \\
\hline
\end{tabular}

Values are mean absolute glucose metabolic rates adjusted for age effects. Significant differences in comparison with normal controls via pairwise comparisons with a Tukey-Kramer adjustment for multiple comparisons: ${ }^{3} p<0.0001,{ }^{c} p<0.01,{ }^{d} p<0.05,{ }^{n s}=$ not significant. dOPCA = dominantiy inherited olivopontocerebellar atrophy; sOPCA $=$ sporadic olivopontocerebellar atrophy; $\mathrm{MSA}=$ multiple system atrophy.

tween diagnosis groups $(p<0.0001)$ and evidence for age effects $(p<0.001)$ but no other significant effects.

The significant effects found berween regions and differences in the effects of diagnosis groups indicated that differences in metabolic rates between diagnosis groups must be examined for each region individually via a one-way ANOVA on diagnosis group. The evidence of differences in age effects by region and an overall age effect require that age be incorporated in the univariate analysis with an ANCOVA by region.

Separate univariate analysis for each region (including the factor diagnosis and the covariate age) demonstrated that age effects were significant in the brainstem $(p=0.012)$, cerebellar vermis $(p=0.005)$, cerebellar hemispheres $(p=0.007)$, and thalamus $(p=0.017)$. In the caudate nucleus there was some indication of a trend with age $(p=0.06)$, but no evidence for an age effect was found in the putamen $(p>0.10)$. To provide a common basis for comparison, subsequent analyses of normalized metabolic rates by diagnosis group included an adjustment for age in all regions.

Very strong evidence was found for overall differences in diagnosis groups in brainstem, cerebellar vermis, and cerebellar hemispheres ( $p<0.0001$ in each). Strong evidence for overall differences in diagnosis groups was also found in the putamen $(p=0.01$ ). No evidence was found for differences in the caudate nucleus or thalamus $(p>0.10)$. To investigate these differences further, pairwise comparisons among mean ICMRglc values were examined.

After controlling for multiple comparisons, we found that in MSA, normalized ICMRglc was markedly and significantly decreased relative to normals in the brainstem, cerebellar vermis, and cerebellar hemispheres $(p<0.0001$ in each) and also in the putamen 


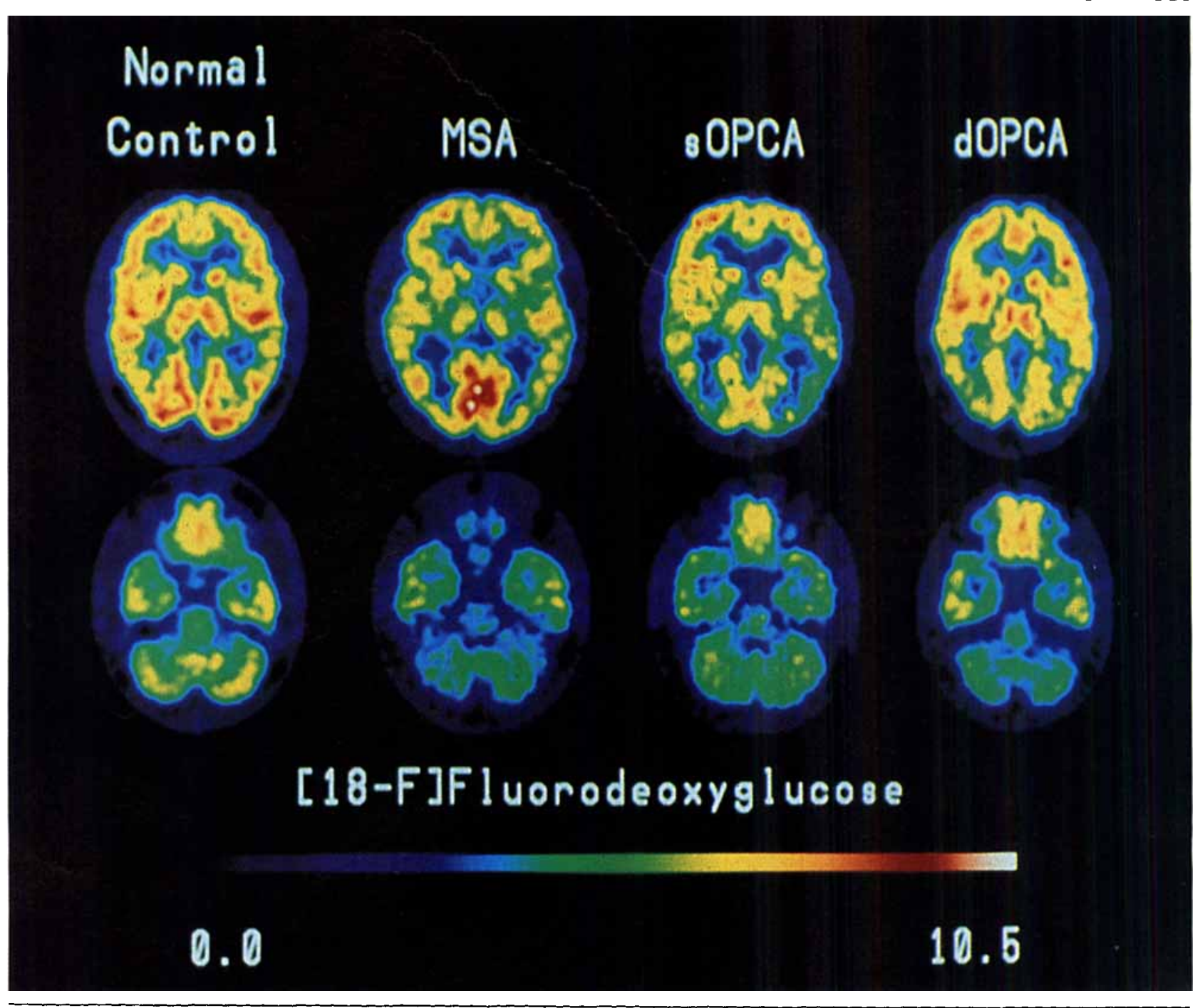

Positron emission tomograpby scans sbowing local cerebral metabolic rate for glucose (lCMRglc) as detected with $\left(^{18} F\right)$ fluorodeoxyglucose in a male normal control subject age 52 (left upper and lower images), a male patient age 61 with multiple system atropby (MSA, upper and lower images second from the left), a male patient age 46 with sporadic olivopontocerebellar atropby (sOPCA, upper and lower images third from the left), and a male patient age 37 with dominantly inberited olivopontocerebellar atropby (dOPCA, right upper and lower images). All scans in the upper row show borizontal sections at the level of the cerebral cortex, basal ganglia, and thalamus, and all scans in the lower row show horizontal sections at the level of the cerebellum and the base of the temporal and frontal lobes. The color bar indicates the rate of cerebral glucose utilization for all scans illustrated expressed as milligrams per 100 grams per minute extending from 0.0 to 10.5. Note the decreased ICMRglc in the brainstem and cerebellum of all 3 patients in comparison with the normal control subject. There is decreased lCMRglc also in the cerebral cortex and basal ganglia of the patients with MSA and SOPCA but not dOPCA. $(p<0.01)$ (Table 4$)$. In both sOPCA and dOPCA, ICMRglc was decreased in the brainstem, cerebellar vermis, and cerebellar hemispheres $(p<0.0001$ in each). No statistically significant differences between MSA and DOPCA were found.

\section{Neuropatbological Findings}

Four of the 14 patients with MSA in the present study have died and come to neuropathological examination. The diagnosis of MSA was confirmed in 3 of these patients by the marked degenerative changes, including loss of neurons and gliosis, in the inferior olives, pons, cerebellum, substantia nigra, and posterior lateral aspects of the putamen. There was no involvement of the anterior thalamus, caudate nucleus, hypothalamus, or the frontal, parietal, temporal, and occipital regions of the cerebral cortex. The fourth patient survived cardiac arrest and lingered in a vegetative state for 4 weeks before succumbing. The preexistent neuropathological changes in the forebrain could not be estab- 
Table 4. Local Cerebral Metabolic Rates for Glucose Normalized to the Cerebral Cortex

\begin{tabular}{|c|c|c|c|c|}
\hline Structure & $\begin{array}{l}\text { Normal } \\
\text { Controls } \\
(\mathrm{n}=51)\end{array}$ & $\begin{array}{l}\text { dOPCA } \\
(\mathrm{n}=19)\end{array}$ & $\begin{array}{l}\text { sOPCA } \\
(\mathrm{n}=32)\end{array}$ & $\begin{array}{l}\text { MSA } \\
(\mathrm{n}=24)\end{array}$ \\
\hline Brainstem & 0.86 & $0.71^{\mathrm{a}}$ & $0.73^{\mathrm{a}}$ & $0.68^{a}$ \\
\hline $\begin{array}{l}\text { Cerebellar } \\
\text { vermis }\end{array}$ & 0.91 & $0.72^{\mathrm{a}}$ & $0.71^{\mathrm{a}}$ & $0.73^{\mathrm{a}}$ \\
\hline $\begin{array}{l}\text { Cerebellar } \\
\text { hemispheres }\end{array}$ & 0.98 & $0.76^{a}$ & $0.77^{\mathrm{a}}$ & $0.71^{\mathrm{a}}$ \\
\hline Thalamus & 1.26 & $1.24^{\mathrm{ns}}$ & $1.26^{\mathrm{ns}}$ & $1.24^{\mathrm{ns}}$ \\
\hline Caudate nuclei & 1.16 & $1.13^{\mathrm{ns}}$ & $1.18^{\mathrm{ns}}$ & $1.18^{\mathrm{ns}}$ \\
\hline Putamen & 1.26 & $1.25^{\mathrm{ns}}$ & $1.24^{\mathrm{ns}}$ & $1.17^{c}$ \\
\hline
\end{tabular}

Values are mean cerebral glucose metabolic rates normalized to cerebral cortex and adjusted for age effects. Significant differences in comparison to normal controls via pairwise comparisons with Tukey-Kramer adjustment for muitiple comparisons: ${ }^{a} p<0.0001$, ${ }^{c} p<0.01,{ }^{n s}=$ not significant.

$\mathrm{dOPCA}=$ dominantly inherited olivopontocerebellar atrophy; sOPCA $=$ sporadic olivopontocerebellar atrophy; $\mathrm{MSA}=$ multiple system atrophy.

lished because of extensive hypoxic-ischemic tissue damage, but degenerative and atrophic changes were found in the inferior olives, pons, and cerebellum.

\section{Discussion}

We found in all three groups of patients, MSA, sOPCA and DOPCA, a marked and significant decrease of absolute ICMRglc in the brainstem and cerebellar vermis and hemispheres. MSA patients showed additionally a marked and significant decrease in the putamen, and a mild but significant decrease in the thalamus and cerebral cortex, but no significant change in the caudate nucleus. Patients with SOPCA showed, in addition, a mild but significant decrease in the putamen, thalamus, and cerebral cortex and no change in the caudate nucleus. Patients with dOPCA showed no significant differences in other structures, including the thalamus, putamen, caudate nucleus, and cerebral cortex. Thus, distinctive regional patterns of decreased ICMRglc are found in all three disorders, with more similarity between sOPCA and MSA than between DOPCA and either MSA or SOPCA.

Comparison of $1 \mathrm{CMRglc}$ in the putamen among the three groups of diseases disclosed (1) a large and significant reduction in $M S A$, (2) a significant reduction in SOPCA but not as large as that seen in MSA, and (3) no significant change in dOPCA. Since patients with sOPCA can progress to develop MSA, it is possible that the moderate reduction of ICMRglc in the putamen in these patients may represent the development of neuropathological changes typical of MSA, although clinical signs diagnostic of MSA were not detectable at the time of study.

When the ICMRglc data were normalized to the ce- rebral cortex, all three groups of patients showed a marked and significant decrease in the brainstem and cerebellum. In MSA patients a mild but significant decrease was found also in the putamen. In SOPCA and dOPCA patients, no other regions showed significant changes. These findings reflect the severe involvement of the brainstem and cerebellum in all three disorders and the greater involvement of the putamen in MSA than in the other two entities. None of the other regions that were significantly affected in the analysis of absolute data in SOPCA and MSA groups showed significant changes in the normalized data because these regions were all mildly affected to about the same degree.

The finding of markedly reduced ICMRglc in the putamen as well as the brainstem and cerebellum in MSA patients is in keeping with the neuropathological changes in this disease $[4,8,9,12,14,15]$ and, to some extent, with previous PET studies [36-38]. Degenerative changes in MSA are prominent in the putamen, substantia nigra, and locus ceruleus and also commonly affect the inferior olives, pons, and cerebellum $[2,4,8]$. Thus, hypometabolism in the putamen, as well as the brainstem and cerebellum, reflects the loss of neurons and synaptic connections in these sites. Many other sites can be affected neuropathologically in MSA, including the cerebral cortex, caudate nucleus, and globus pallidus (especially the external portion), but the thalamus is only rarely involved. Thalamic involvement when present is probably due to deafferentation secondary to degeneration of striatothalamic projections, which is evident in the ansa lenticularis and fasciculus lenticularis. The finding of normal glucose metabolic rates in the caudate nucleus of MSA patients in the present study indicates that this site was not appreciably affected in our cases, but the hypometabolism found in the cerebral cortex suggests that this site is either directly affected or shows the effects of deafferentation. The finding of decreased glucose metabolic rates in the cerebral cortex suggests that PET studies with FDG can demonstrate disturbances not detected with conventional neuropathological techniques.

The finding of significantly reduced ICMRgic in the brainstem and cerebellum in both SOPCA and DOPCA is in keeping with the primary neuropathological process in OPCA, which involves neuronal loss and gliosis within the inferior olives, pons, and cerebellum $[2$, 19-231. The decreased metabolic rates in these structures reflect the reduced numbers of neurons and synaptic connections, though partial volume effects from tissue atrophy may be important. Although many other central nervous system structures can show neuropathological findings in OPCA $[2,19-23]$, the finding of reduced ICMRglc so widely throughout the brain in patients with SOPCA in this study, including the basal 
ganglia, thalamus, and cerebral cortex, is unexpected but may suggest that (1) this disorder affects many more structures in the central nervous system than conventional neuropathological studies have disclosed, (2) the sites showing primary degenerative changes influence other structures through transneuronal effects, or (3) these patients are beginning to develop MSA.

Several investigators have examined ICMRglc in small numbers of MSA patients. De Volder and colleagues [36] studied 7 patients with probable SND compared with 16 neurologically normal control subjects by using FDG and PET. All 7 patients had extrapyramidal symptoms poorly responsive or unresponsive to levodopa, and 2 of the 7 had signs of cerebellar disorder accompanied by autonomic disturbances. Glucose utilization was significantly decreased in the patients with SND in the putamen, caudate nucleus, and frontal regions of the cerebral cortex but not in the thalamus or cerebellum. In the 2 patients with cerebellar symptoms, however, hypometabolism was found within the cerebellum. Moreover, a third patient scanned twice showed clinical signs of cerebellar disorder at the time of the second scan, and only this scan demonstrated cerebellar hypometabolism. Our patients differed from those of De Volder and colleagues [36] in that we had more who had involvement of the cerebellum. Fulham and associates [37] studied 8 MSA patients in comparison with 18 normal control subjects by using FDG and PET and found hypometabolism in the cerebellar vermis, cerebellar hemispheres, striatum, and frontal cortex. Four of their 8 patients had prominent signs of cerebellar disorder. Their findings are similar to those in our MSA patients. Eidelberg and co-workers [38] reported finding hypometabolism in the caudate nucleus and putamen of 10 patients with striatonigral degeneration in comparison with 10 normal control subjects and 20 patients with Parkinson's disease by using FDG and PET. They found no metabolic changes in other cortical or subcortical regions.

This study was supported in part by grants NS15655 and AG08671 from the National Institutes of Health.

\section{References}

1. Polinsky RJ. Multiple system atrophy. Clinical aspects, pathophysiology and treatment. Neurol Clin 1984:2:487-498

2. Oppenheimer SE. Diseases of the basal ganglia, cerebellum and motor neurons. In: Humes AJ, Corsellis JAN, Duchen IW, eds. Greenfield's neuropathology. 4th ed. New York: Wiley, 1984:699-747

3. Cohen J, Low P, Fealey R, et al. Somatic and autonomic function in progressive autonomic failure and multiple system atrophy. Ann Neurol 1987;22:692-699

4. Quinn N. Multiple system atrophy-the nature of the beast. J Neurol Neurosurg Psychiatry 1989;(spec suppl):78-89)

5. Sandroni P, Ahlskog JE, Fealey RD, Low PA. Autonomic involvement in extrapyramidal and cerebellar disorders. Clin Auton Res 1991;1:147-155

6. Izume $\mathrm{K}$, Inoue $\mathrm{N}$, Shirabe $\mathrm{T}$, et al. Failed levodopa therapy in striato-nigral degeneration. Lancet 1971;1:1955

7. Rajput AH, Kazi KH, Rozdilsky B. Striatonigral degeneration response to levodopa therapy. J Neurol Sci 1972;16:331-341

8. Kume A, Takahashi A, Hashizume Y, Asai J. A histometrical and comparative study on Purkinje cell loss and olivary nucleus cell loss in multiple system atrophy. J Neurol Sci 1991;101: $178-186$

9. Takei Y, Mirra SS. Striatonigral degeneration: a form of multiple system atrophy with clinical Parkinsonism. In: Zimmerman HM, ed. Progress in neuropathology. New York: Grune and Stratton, 1973:217-251

10. Borit A, Rubinstein LJ, Urich $\mathrm{H}$. The striatonigral degenerations: putaminal pigments and nosology. Brain 1975;98:101112

11. Gibb WRG. Accuracy in the clinical diagnosis of parkinsonian syndromes. Postgrad Med J 1988;64:345-351

12. Fearnley JM, Lees AJ. Striatonigral degeneration. A clinicopathological study. Brain 1990;113:1823-1842

13. Bannister R, Oppenheimer DR. Degenerative diseases of the nervous system associated with autonomic failure. Brain 1972; 95:457-474

14. Sung JH, Mastri AR, Segal E. Pathology of Shy-Drager syndrome. J Neuropathol Exp Neurol 1979;38:353-368

15. Spokes EGS, Bannister R, Oppenheimer DR. Multiple system atrophy with autonomic failure. J Neurol Sci 1979;43:59-82

16. Petito CK, Hart MN, Porro RS, Earle KM. Ultrastructural studies of olivopontocerebellar atrophy. J Neuropathol Exp Neurol $1973 ; 32: 503-522$

17. Landis DMD, Rosenberg RN, Landis SC, et al. Olivopontocerebellar degeneration. Clinical and ultrastructural abnormalities. Arch Neurol 1974;31:295-307

18. Chokroverty S. Autonomic dysfunction in olivopontocerebellar atrophy. In: Duvoisin RC, Plaitakis A, eds. The olivopontocerebellar atrophies. New York: Raven, 1984:105-141

19. Koeppen $\mathrm{AH}, \mathrm{Baron} \mathrm{KD}$. The neuropathology of olivopontocerebellar atrophy. In: Duvoisin RC, Plaitakis A, eds. The olivopontocerebellar atrophies. New York: Raven, 1984;13-38

20. Gilman S, Bloedel JR, Lechtenberg R. Disorders of the cerebelIum. Philadelphia: Davis, 1981

21. Harding AE. The hereditary ataxias and related disorders. London: Churchill Livingstone, 1984

22. Eadie MJ. Olivo-ponto-cerebellar atrophy (Dejerine-Thomas type). In: Vinken PJ, Bruyn GW, eds. Handbook of clinical neurology. Amsterdam: North-Holland, 1975:415-431

23. Eadie MJ. Olivo-ponto-cerebellar atrophy (Menzel type). In: Vinken PJ, Bruyn GW, eds. Handbook of clinical neurology. Amsterdam: North-Holland, 1975:433-449

24. Gilman S. Markel DS, Koeppe RA, et al. Cerebellar and brainstem hypometabolism in olivopontocerebellat atrophy detected with positron emission tomography. Ann Neurol 1988;24:223230

25. Rosenthal G, Gilman S, Koeppe RA, et al. Motor dysfunction in olivopontocerebellar atrophy is related to cerebral metabolic rate studied with positron emission tomography. Ann Neurol 1988;24:414-419

26. Kluin KJ, Gilman S, Markel DS, et al. Speech disorders in olivopontocerebellar atrophy correlate with positron emission tomography findings. Ann Neurol 1988;23:547-554

27. Gilman S, Koeppe RA, Junck I, et al. Extensive cerebral hypometabolism in multiple system atrophy detected with $\left[{ }^{18} \mathbf{F}\right\}$ fluorodeoxyglucose and positron emission tomography. Neurology 1992:42(suppl 3):398-399

28. Savoiardo M, Strada L, Girotti $F$, et al. MR imaging in progres- 
sive supranuclear palsy and Shy-Drager syndrome. J Compur Assist Tomogr 1989;13:550-560

29. Savoiardo M, Strada L, Girotti F, et al. Olivopontocerebellar atrophy: MR diagnosis and relationship to multisystem atrophy. Radiology 1990;174:693-696

30. Brooks DJ. Reply to the diagnosis of multiple system atrophy by T Caraceni. Ann Neurol 1991;29:690 (Letter)

31. McLeod JG, Tuck RR. Disorders of the autonomic nervous system: Part 2. Investigation and treatment. Ann Neurol 1987; 21:519-529

32. Kluin KJ, Foster NL, Berent S, Gilman S. Perceptual analysis of speech in progressive supranuclear palsy. Neurology 1993; 43:563-566

33. Hamacher K, Coenen HH, Stocklin G. Efficiency stereospecific synthesis of NCA 2-[ $\left.{ }^{18} \mathrm{~F}\right\}$-fluoro-2-deoxy-D-glucose using aminopolyether supported direct nucleophilic substitution. J Nucl Med 1986;27:235-238
34. Hutchins GD, Holden JE, Koeppe RA, et al. Alternative approach of single-scan estimation of cerebral glucose metabolic rate using glucose analogs, with particular application to ischemia. J Cereb Blood Flow Metab 1984;4:35-40

35. Hochberg Y, Tamhane AC. Multiple comparison procedures. New York: Wiley, 1987:91-92

36. De Volder AG, Francart J, Laterre C, et al. Decreased glucose utilization in the striatum and frontal lobe in probable striatonigral degeneration. Ann Neurol 1989;26:239-247

37. Fulham MJ, Dubinsky RM, Polinsky RJ, et al. Computed tomography, magnetic resonance imaging and positron emission tomography with $\left[{ }^{18} \mathrm{~F}\right]$ fluorodeoxyglucose in multiple system atrophy and pure autonomic failure. Clin Auton Res 1991;1: $27-36$

38. Eidelberg D, Takikawa S, Moeller JR, et al. Striatal hypometabolism distinguishes striatonigral degeneration from Parkinson's disease. Ann Neurol 1993;33:518-527 